\title{
ON THE ACOUSTICS OF ROCKET COMBUSTORS EQUIPPED WITH QUARTER WAVE ABSORBERS
}

\author{
M. Oschwald and M. Marpert \\ DLR Lampoldshausen Langer Grund \\ D 74239 Hardthausen, Deutschland
}

The acoustic resonance spectrum and the dissipation of specific modes in combustors equipped with absorbers have been investigated experimentally and numerically. It is found that the application of absorber rings to a combustor changes its resonance behavior significantly. Based on the acoustic fields obtained by three-dimensional (3D) modal analysis, the damping behavior for modes is predicted and compared to measurements. There is a good agreement between prediction and experimental data with respect to the general trend of the dependence of damping on the absorber length. However, the experimentally determined dissipation rates are significantly larger than the predicted values.

\section{INTRODUCTION}

The application of quarter-wave absorbers to combustion chambers is a wellknown approach to increase the dissipation rate of acoustic energy so as to increase the stability margins of a rocket motor with respect to high frequency instabilities [1-3]. Usually, both resonance volumes - the absorber as well as the combustor - are treated as two acoustically independent resonators. The eigenfrequency $f_{A}$ of the absorber is then tuned in a way that it is in resonance to the eigenfrequency of the combustion chamber $f_{C}$ that should be damped. For a half-open resonator, its resonance frequency $f_{A}$ is

$$
f_{A}=\frac{c}{4 L}
$$

and the resonance frequency $f_{C}$ of a cylindrical chamber is [2]

$$
f_{C}=\alpha_{n m} \frac{c}{2 \pi R}
$$

This is an Open Access article distributed under the terms of the Creative Commons Attribution-Noncommercial License 3.0, which permits unrestricted use, distribution, and reproduction in any noncommercial medium, provided the original work is properly cited. 
For the first tangential mode, it is $\alpha_{11}=1.841$ and for a cavity tuned to the 1T-mode, a length $L_{A}$ of

$$
L_{A}=0.853 R
$$

is obtained, and for the 2T-mode with $\alpha_{12}=3.054$ the length of a tuned cavity is

$$
L_{A}=0.514 R
$$

This basic approach does not take into account that the coupled system of absorbers and combustor cannot be regarded as the sum of two acoustically independent resonators. Generally, the resonance frequencies of the coupled system are matching neither the eigenfrequencies of the combustor nor that of the absorber [4]. With increasing absorber length, the resonance frequencies of the coupled system are decreasing and the symmetry of the modes in the combustion chamber may exhibit significant changes. Furthermore, it has been shown experimentally [4] as well as numerically [4, 5] that maximum damping of a quarter-wave absorber mounted to a combustor is not obtained at an absorber length $L_{A}$ determined by Eq. (2) but at larger lengths. Again, this can be understood based on the acoustic coupling of combustor and absorber.

Following what has been learned from a single absorber mounted to a combustor, the work has been extended to absorber rings [6]. Basic properties of the resonance spectrum of a chamber equipped with absorber rings have been analyzed based on modal analysis of two-dimensional (2D) models. As in the case of one absorber, all resonance frequencies are decreasing with increasing absorber lengths for a combustor with an absorber ring. Especially for absorbers tuned to the frequency of the 1T-mode according to Eq. (2), the spectrum exhibits resonances significantly different from that for cylindrical resonators. The qualitative damping behavior of the absorber ring as a function of the absorber length has been discussed based on the numerically determined acoustic velocity fields of the eigenmodes. For the 1T-mode, this qualitative analysis predicted for an absorber ring a continuously increasing damping with increasing absorber length, specifically there has been found no maximum in the damping rate for a specific absorber length.

This work presents the results of an improved analysis of the resonance frequencies and damping behavior for absorber rings. The modal analysis is done based on a 3D model in order to allow a quantitative comparison with a reference experiment. Searby et al. have published a method to derive quantitative values for the damping rate from the acoustic velocity fields of eigenmodes [5]. This method is used in this work to determine the damping behavior of the absorber rings and the predictions are compared with experimental data. 


\section{EXPERIMENTAL SETUP}

\subsection{Combustion Chamber and Absorber Ring}

Experiments have been done with an absorber ring used during the development of steam generators for the high altitude simulation test bench P4.1 in Lampoldshausen [7].

A basic sketch of the steam generator is shown in Fig. 1. The combustor has a diameter of $22 \mathrm{~cm}$. The injection plate is replaced by a face plate on which a loadspeaker and a microphone are mounted. The length of the combustor can be reduced in the experiments by an optional closure plate in order to shift the resonance frequencies of the longitudinal modes to higher values. The longitudinal modes thus are not interfering with the frequencies of the transversal modes of interest in this investigation. The application of the closure plate does not limit the statements done in this paper with respect to the application of absorber rings to combustors.

The absorber ring is shown in Fig. 2. Forty two absorbers are mounted around the circumference of the ring. The angular distance between neighboring absorbers is constant with the exception of three positions where the option to mount sensors has been foreseen. Thus, the circumferential distribution of the absorbers is not perfectly symmetric. The diameter of the absorbers is $8 \mathrm{~mm}$, each absorber can be individually tuned to a specific length. The maximum value that could be adjusted with the given hardware was $L_{A} / R_{C}$ $=0.8$. In the experiments presented here, all absorbers were tuned to the same length.

The acoustic properties of the combustor equipped with the absorber ring have been determined with the chamber filled with air at ambient temperature. The measured resonance frequencies are therefore smaller than typical frequencies in hot fire tests by about a factor of 4 .

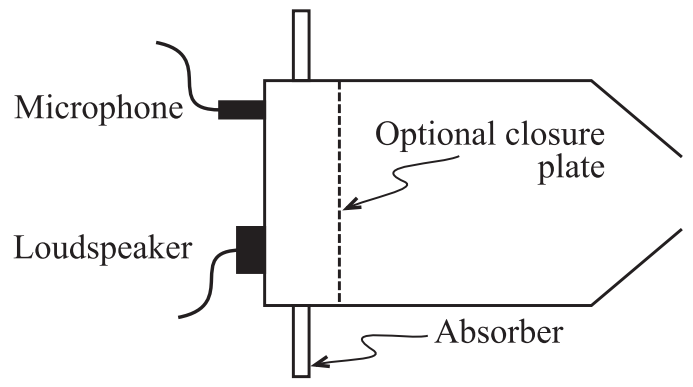

Figure 1 Sketch of the steam generator combustion chamber 


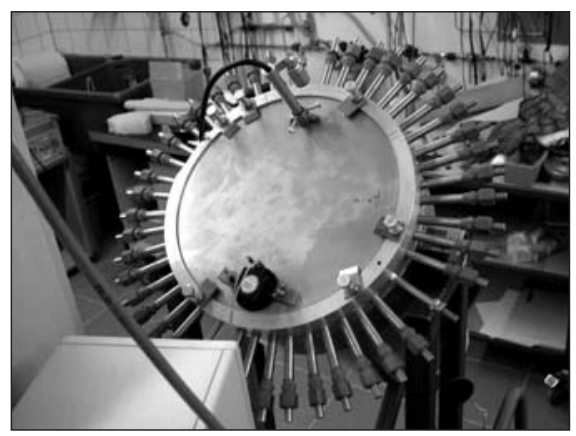

Figure 2 Absorber ring mounted to the steam generator

\subsection{Instrumentation and Data Reduction}

Acoustic oscillations are excited by a loadspeaker mounted in the face plate as seen in Fig. 1. The loudspeaker is operated on a single frequency to excite a specific mode. Alternatively, a synthetic white noise signal is used to excite all resonances simultaneously.

After some time, the loudspeaker is switched off and the decay of the acoustic wave is recorded by a microphone mounted on the faceplate opposite to the loudspeaker (see Figs. 1 and 2). A Fourier transform of the decaying signal provides the resonance frequency. From the full width at half maximum $\Delta f$ of the resonance profile, the damping of the acoustic energy is derived [4]. The decay rate $\sigma$ of the acoustic pressure is then determined from $\sigma=\pi \Delta f$.

\section{NUMERICAL ANALYSIS}

\subsection{Determination of Eigenfrequencies}

The geometry of the steam generator with the absorber ring has been mapped on a 3D-mesh. ANSYS was used to determine numerically the acoustic eigenmodes of the resonance volume. The analysis has been done for the combustor with its length reduced by the closure plate. The results provide the resonance frequencies as well as the spatial distributions of the acoustic pressure and velocity fields of the eigenmodes. From the analysis of the $2 \mathrm{D}$ problem [6], it has been found that the resonance frequencies for a combustor with an absorber ring are organized in a specific way. For an absorber ring with 42 absorbers tuned to the $1 \mathrm{~T}$ resonance, the first 47 transversal eigenmodes have to be determined to catch all modes with frequencies up to the $1 \mathrm{R}$-frequency. For this reason, the 
first 64 eigenmodes have been determined for absorber lengths $L_{A} / R_{C}$ ranging from 0 to 2.0 .

\subsection{Evaluation of Damping}

In [4], a qualitative approach has been used to extract information on the damping behavior of absorber rings from the results of the 2D-modal analysis.

Searby et al. $[5,8]$ recently have proposed a hybrid approach to determine the acoustic damping due to viscous and thermal dissipation. From numerically obtained acoustic velocity fields of eigenmodes and analytical solutions for the dissipation in oscillating shear layers, they determined the damping of the eigenmodes. A comparison of the hybrid approach with analytical solutions available for simple geometries and with results from computational fluid dynamics (CFD) calculations for more complex configurations has resulted in a good agreement between the methods [5].

The hybrid method has been implemented in the authors' code and quantitative predictions of the damping rate $\sigma$ are thus obtained for the eigenmodes determined with ANSYS.

\section{RESULTS}

\subsection{Resonance Frequencies}

The resonance spectrum for the absorbers tuned to zero length, i.e., for a cylindrical resonator together with the prediction for the eigenfrequencies according to Eq. (1) is shown in Fig. 3 and a very good agreement is found.

The results as obtained by the experiment and the numerical modal analysis are plotted for modes $m=1$ to 5 in Fig. 4 . For a cylinder without absorbers, the tangential modes are twofold degener-

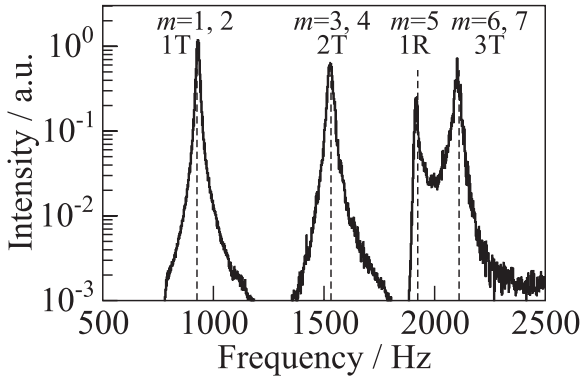

Figure 3 Resonance spectrum for the combustor without absorbers $\left(L_{A} / R_{C}=0\right)$ ate. Thus, the first five modes cover the modes with $1 \mathrm{~T}(m=1,2), 2 \mathrm{~T}(m=3,4)$ and 1R-symmetry $(m=5)$ in the limit $L_{A} \rightarrow 0$. Due to the mounting of the absorbers, the rotational symmetry is not perfect and the frequencies predicted by the numerical analysis for the 


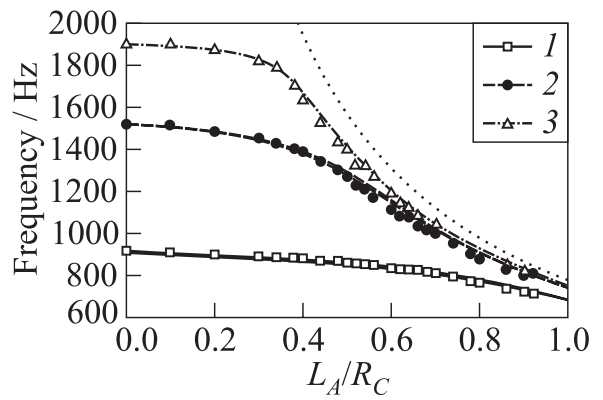

Figure 4 Experimental (signs) and numerical (curves) results for the resonance frequencies of the first 5 modes of a cylinder with absorber ring as function of absorber length: $1-1 \mathrm{~T} ; 2-2 \mathrm{~T}$; and $3-1 \mathrm{R}$. Dotted curve refers to $\lambda / 4$

tors with absorber rings where commonly resonances with frequencies near to higher cylinder modes are observed. The problem is resolved when looking at Fig. 5 where the calculated resonance frequencies for the first 64 eigenmodes are plotted.

Now, it can be seen that there are resonances above the quarter wave frequency; however, the first mode with a frequency above the absorber resonance is the mode with $m=43$. The systematics already found in the 2Danalysis [6] of absorber rings is confirmed for the 3D-analysis here: for an

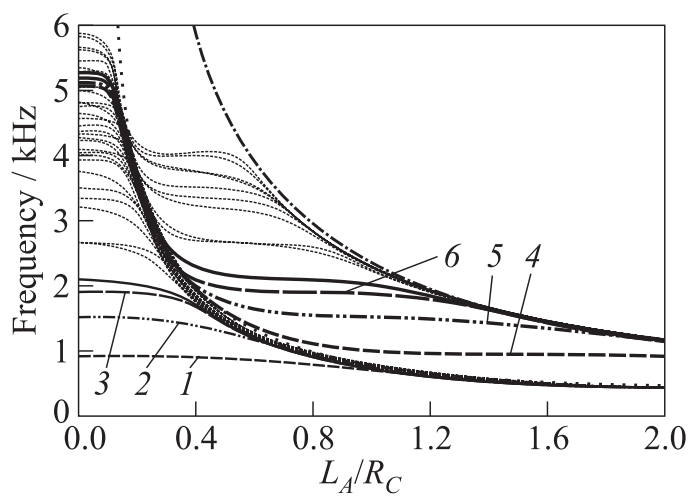

Figure 5 Numerical results of the first 64 resonance frequencies of a cylinder with absorber ring as function of the absorber length: $1-m=1,2(1 \mathrm{~T}) ; 2-m=3,4$ $(2 \mathrm{~T}) ; 3-m=5(1 \mathrm{R}) ; 4-m=43,44(1 \mathrm{~T}) ; 5-m=45,46(2 \mathrm{~T}) ;$ and $6-m=47$ (1R). The dotted curve refers to $\lambda / 4$ and the dash-dotted curve refers to $3 \lambda / 4$ 


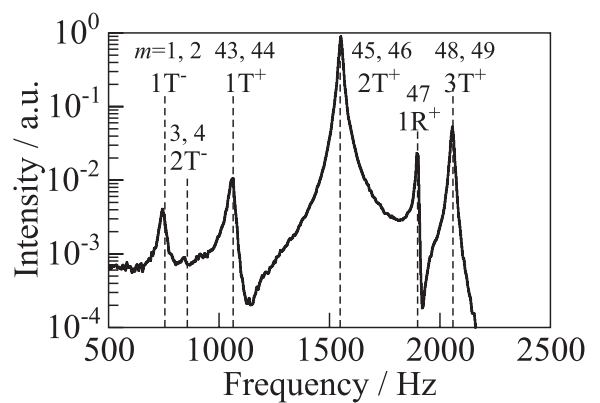

(a)

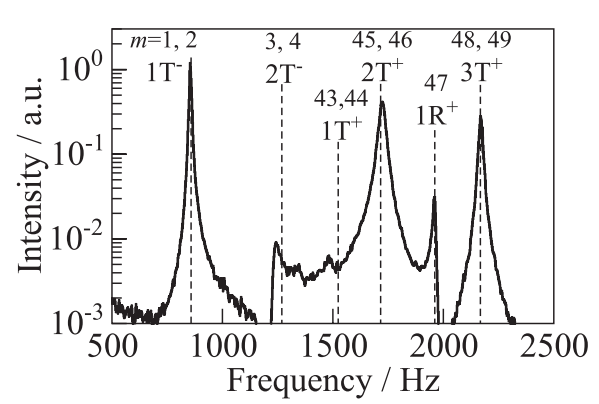

(b)

Figure 6 Resonance spectrum for absorbers tuned to the $1 \mathrm{~T}\left(L_{A} / R_{C}=0.85\right)(a)$ and $2 \mathrm{~T}\left(L_{A} / R_{C}=0.51\right)(b)$ quarter wave frequencies

absorber ring with $n$ absorbers, the first $n$ modes have eigenfrequencies below, modes $m=n+1$ and higher have frequencies above the quarter wave resonance of the absorbers. As a second asymptotic, the $3 \lambda / 4$ resonance appears in Fig. 5.

Figure $6 a$ presents the resonance spectrum for the specific case where the length of the absorbers is tuned to the $1 \mathrm{~T}$ cylinder mode according to Eq. (2). The resonance frequencies as predicted by the 3D modal analysis are included in the plot and show a very good agreement with the experiment. Although the spectrum looks similar to the spectrum of a cylindrical resonator, there are significant differences. Near the $1 \mathrm{~T}$ frequency, now two peaks are seen, the peak corresponding to the modes with the number $m=1,2$ is shifted to a lower frequency as compared for $L_{A}=0$, the peak for modes $m=43,44$ is at a higher frequency. The symmetry of the pressure fields of the modes $m=1,2$, and 43 , 44 as obtained by the modal analysis exhibit a 1T-symmetry in the cylindrical chamber and this justifies to label these modes $1 \mathrm{~T}^{-}$and $1 \mathrm{~T}^{+}$, respectively. The predicted resonance for modes $m=3,4$ is rather not seen in the experimental spectrum. In Fig. 5, it can be seen that for $L_{A} / R_{C}=0.85$, the modes $m=3,4$ are very near to the $\lambda / 4$-resonance.

No peaks corresponding to the modes $m=5$ to $m=42$, all very near to the absorber resonance, are seen in Fig. $6 a$. It is a general observation that modes with a resonance near to an absorber resonance are strongly damped and therefore not present in the experimental spectra.

Further, resonance peaks near to the 2T-, 1R-, and 3T-frequencies are found. However, as seen in Fig. 5, these resonances do not origin from the corresponding cylinder modes in the limit $L_{A} / R_{C} \rightarrow 0$. The modal analysis confirms again that these modes with resonance frequencies similar to that of cylinder modes show the corresponding symmetry. As in the case for the modes with $1 \mathrm{~T}$ sym- 


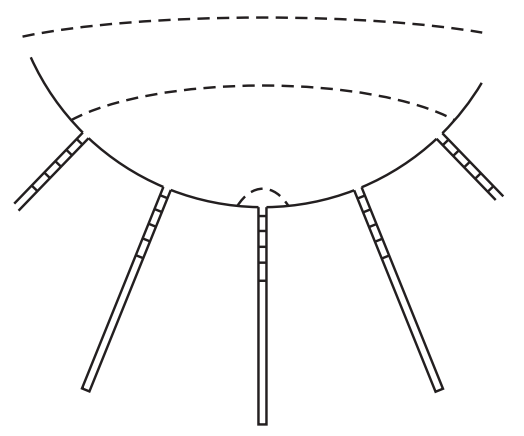

(a)

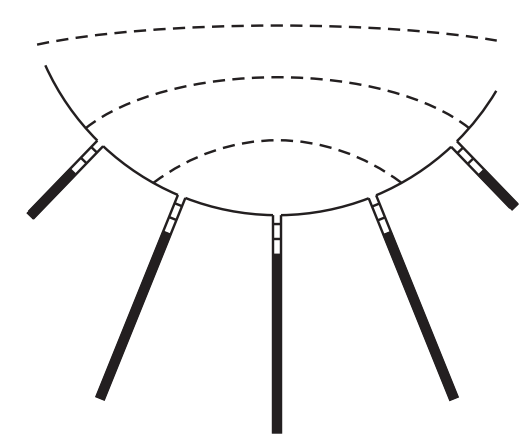

(b)

Figure 7 Zoom on the acoustic pressure field for the $1 \mathrm{~T}^{-}(a)$ and $1 \mathrm{~T}^{+}(b)$ modes in the absorbers

metry, these resonances are labeled according to their symmetry as $2 \mathrm{~T}^{+}, 1 \mathrm{R}^{+}$, and $3 \mathrm{~T}^{+}$. This classification of modes works only for the limited range of absorber lengths $L_{A} / R_{C} \approx 0.4-1.1$. As in practice absorber lengths are adjusted in this range, this classification may be useful to understand the observed acoustic resonances.

The resonance spectrum for the absorbers tuned to the $2 \mathrm{~T}$ cylinder mode according to Eq. (3) is shown in Fig. 6b. Peaks are found near to the 1T, 1R, and $3 \mathrm{~T}$ cylinder resonances. With the help of the modal analysis, the peaks can be attributed to the $1 \mathrm{~T}^{-}, 1 \mathrm{R}^{+}$, and $3 \mathrm{~T}^{+}$modes, respectively. There is no resonance peak visible at the $2 \mathrm{~T}$ frequency but instead, the $2 \mathrm{~T}^{-}$resonance at lower frequency and the $2 \mathrm{~T}^{+}$resonance at higher frequency as compared to the frequency of the $2 \mathrm{~T}$ cylinder mode can be identified. In between, the $1 \mathrm{~T}^{-}$ resonance is seen with small amplitude in agreement to its vicinity to the $\lambda / 4$ resonance of the absorbers.

The resonance volume of a combustor with an absorber ring is composed of the cylindrical combustor volume and the attached tubes of the absorbers. Although the symmetries of the $1 \mathrm{~T}^{-}$and $1 \mathrm{~T}^{+}$modes in the combustion chamber are similar, the pressure fields of both modes exhibit an important difference in entrance area of the absorbers.

In Fig. 7, a zoom of the absorber entrance areas is reproduced from [6]. The $1 \mathrm{~T}$-symmetry of the pressure field in the cylindrical part of the resonance volume is clearly seen for both modes. In the absorber entrance area, the $1 \mathrm{~T}^{+}$mode has a pressure node which is not present for the $1 \mathrm{~T}^{-}$mode. As the main contribution to dissipation is due to the characteristics of the acoustic modes in this region, it can be assumed that this results in a different performance of the absorber ring with respect to the damping of the $1 \mathrm{~T}^{-}$and $1 \mathrm{~T}^{+}$modes. This will be discussed in the following subsection. 


\subsection{Damping}

The damping coefficients $\sigma$ for the amplitude of the acoustic pressure derived with the hybrid method for the modes $1 \mathrm{~T}^{-}$and $1 \mathrm{~T}^{+}$are presented in Figs. 8 and 9 , respectively. The damping coefficients for the modes $2 \mathrm{~T}^{-}$and $2 \mathrm{~T}^{+}$are shown in Fig. 10. Near to the $\lambda / 4$ and $3 \lambda / 4$ resonances of the absorbers, there are many modes with similar frequencies (see Fig. 5). In this case, the demand for accuracy of the modal analysis is extremely high and the identity of the modes is hard to determine. For that reason, damping values are only shown for absorber lengths were an unambiguous assignment of the modes could be done. The damping values obtained by experiments are included in the figures. For very strong damping the resonance peak cannot be analyzed for line width any more. Experimental values are therefore only available when the resonance peak shows sufficient signal-to-noise ratio.

For the $1 \mathrm{~T}^{-}$mode (see Fig. 8), a similar behavior is found as for the case of a combustor with one absorber [4]. The maximum damping is obtained at about $L_{A} / R_{C} \approx 1.4$, an absorber length significantly larger than that predicted by Eq. (2). For the predicted length $L_{A} / R_{C}=0.853$, the damping varies with the absorber length, and thus for a tuned absorber ring, the damping is sensitive to variations of the sound velocity. The behavior of $\sigma$ as a function of the absorber length is quite different for the $1 \mathrm{~T}^{+}$mode (see Fig. 9). The mode is strongly damped for small absorber lengths and damping decreases with $L_{A}$. The optimization of damping for both modes at the same time is thus not possible.

The first experimental data obtained for the $1 \mathrm{~T}^{-}$-mode delivered damping values which were about a factor of 5 higher than the prediction of the hybrid

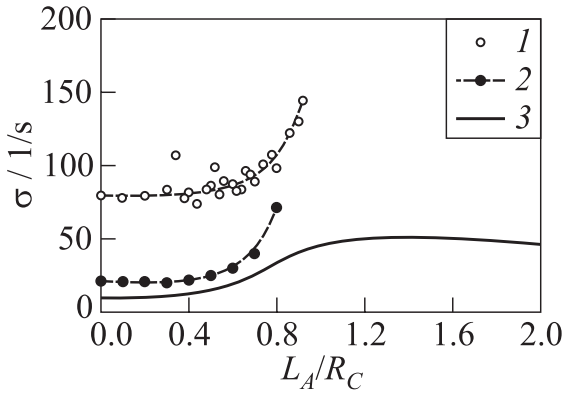

Figure 8 Experimental damping values for the mode $m=1\left(1 \mathrm{~T}^{-}\right)$for the chamber with thin (1) and thick (2) face plates and predictions from the hybrid method (3)

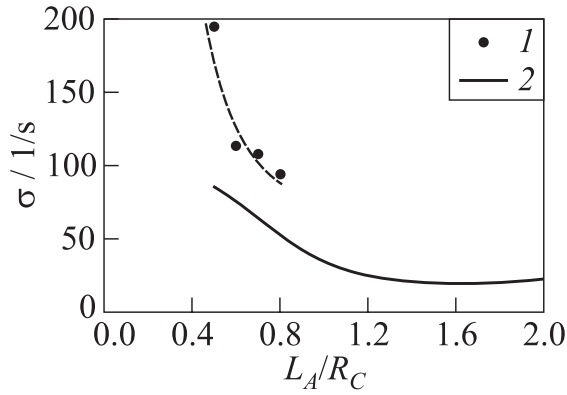

Figure 9 Experimental damping values for the mode $m=44\left(1 \mathrm{~T}^{+}\right)$for the chamber with thick face plates (1) and predictions from the hybrid method (2) 


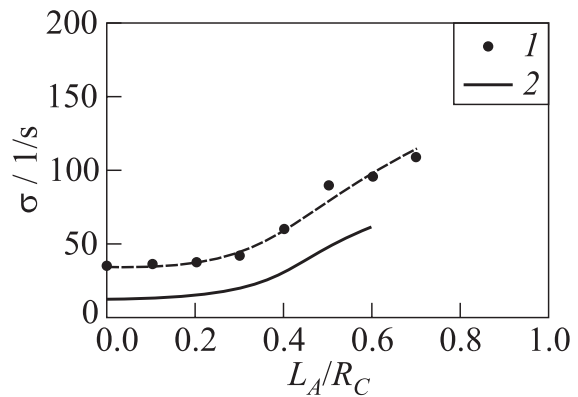

(a)

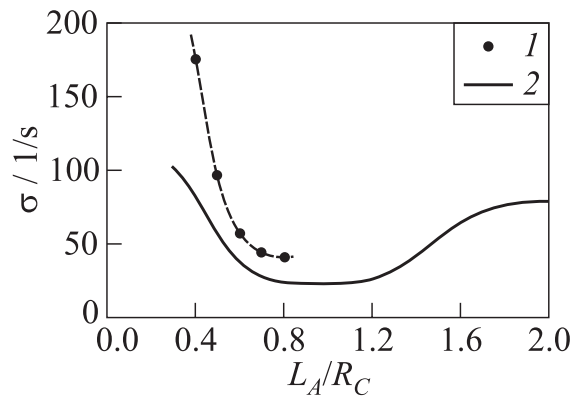

(b)

Figure 10 Experimental damping values for the modes $m=3\left(2 \mathrm{~T}^{-}\right)(a)$ and $m=46$ $\left(2 \mathrm{~T}^{+}\right)(b)$ for the chamber with thick face plates (1) and predictions from the hybrid method $(2)$

method. Two possible explanations for this huge discrepancy have been identified: first, the small thickness of the faceplates which could be bended by the acoustic pressure and thus contribute to the dissipation of acoustic energy, and second, an insufficient sealing between faceplates and absorber ring. A second set of faceplates has been manufactured, thick enough to be rigid, and care was taken to guarantee an efficient sealing between the mechanical parts when mounting together.

With the thick faceplates, the damping determined experimentally is significantly smaller than the data obtained with the thin faceplate. As can be seen in Fig. 8, with the thick faceplates, the data are still a factor of 2 higher than the predictions. One possible explanation could be that the surface of one faceplate is not perfectly flat due to the mounting ports for the loudspeaker and the microphone. Despite the uncertainty about the absolute value for the damping, the experiments clearly confirm the general behavior of the absorber performance as a function of the absorber length. The experimental damping values shown for the $1 \mathrm{~T}^{+}-, 2 \mathrm{~T}^{-}$-, and $\mathrm{T}^{+}$-modes have all been measured with the thick faceplate.

The damping for the modes $2 \mathrm{~T}^{-}$and $2 \mathrm{~T}^{+}$as a function of the absorber length $L_{A}$ follows the same phenomenology. The absorber ring damps the $2 \mathrm{~T}^{-}$ mode more efficiently with increasing absorber length (see Fig. 10a) whereas the damping of the $2 \mathrm{~T}^{+}$mode is decreasing with increasing absorber length (see Fig. 10b). Again, an optimization of damping for both modes at the same time is not possible. For larger absorber lengths when the resonance frequency of the $2 \mathrm{~T}^{+}$mode approaches the $3 \lambda / 4$ frequency, damping starts to increase again. Similar to the 1T-modes, the damping coefficients obtained experimentally for the modes with $2 \mathrm{~T}$-symmetry are systematically higher than the predictions derived with the hybrid method. The general trend of damping as function of absorber length is consistent between experiment and model. 


\section{CONCLUDING REMARKS}

The experimental as well as numerical work clearly shows that the acoustic coupling of an absorber ring to a combustion chamber has a significant influence on the resonance behavior of the coupled system. The eigenspectra with absorber ring differ systematically from that of a combustor without absorbers. The resonance frequencies decrease with absorber length and approach asymptotically the resonance frequencies of the half-open absorber. As a general behavior, it has been found that for a combustor equipped with absorbers with their length tuned to the frequency of a cylinder mode, a resonance at the frequency of this specific mode does not appear in the resonance spectrum. Instead, two resonances one at lower, the other at higher frequency - appear. The corresponding modes show a similar symmetry in the combustor as the mode to which the absorbers were tuned. However, the mode with the higher frequency exhibits a pressure node at the absorber entrance while the mode with the lower resonance frequency does not. As a consequence, the damping behavior for these two modes is different and at no absorber length, maximum damping for both resonances can be achieved at the same time.

The acoustic dissipation for a combustor with absorbers has been predicted based on the acoustic fields obtained by the numerical modal analysis. The predictions show a good agreement with the measurements with respect to the trend of the dependence on the absorber length. However, experimentally determined dissipation rates are significantly higher than the predicted ones. One lesson learned in the frame of this work is that small imperfections of the surface of the resonance volume have a significant influence on the damping behavior. The mounting of loudspeaker and microphone required for the measurements did not allow to realize a perfectly smooth surface and may explain the quantitative difference between predictions and measurements.

\section{REFERENCES}

1. Oberg, C. L., T. L. Wong, and W. M. Ford. 1971. Evaluation of acoustic cavities for combustion stabilization. NASA CR 115087.

2. Harrje, D. T., and F. H. Reardon. 1972. Liquid propellant rocket combustion instability. NASA SP-194.

3. Laudien, E., R. Pongratz, R. Pierro, and D. Preclik. 1995. Experimental procedures aiding the design of acoustic cavities. In: Liquid rocket engine combustion instability. Eds. V. Yang and W. Anderson. Progress in astronautics and aeronautics ser. AIAA Inc. 169:377-99.

4. Oschwald, M., Z. Farago, G. Searby, and F. Cheuret. 2008. Resonance frequencies and damping of a cylindrical combustor acoustically coupled to an absorber. J. Propul. Power 24(3):524-33. 
5. Searby, G., M. Habiballah, A. Nicole, and E. Laroche. 2008. Prediction of the efficiency of acoustic damping cavities. J. Propul. Power 24(3):516-23.

6. Oschwald, M., and Z. Farago. 2008. Acoustics of rocket combustors equipped with absorber rings. AIAA Paper No. 2008-5112.

7. Krühsel, G., and K. Schäfer. 2001. Design and development of an ethanol/lox injection head for rocket steam generator $(45 \mathrm{~kg} / \mathrm{s}$ steam) and experimental study of combustion stability. 4th Conference (International) on Green Propellants for Space Propulsion. Nordwijk, Netherlands: ESTEC.

8. Searby, G. 2009. A lightweight method to estimate the linear acoustic damping of arbitrary complex geometries. 3rd European Conference on Aerospace Sciences. Versailles, France. 